\title{
Olivecrona on the development of neurosurgery in the middle of the twentieth century: reflections with the wisdom of today
}

\author{
Theofilos G. Machinis, M.D., and Kostas N. Fountas, M.D., Ph.D. \\ Department of Neurosurgery, The Medical Center of Central Georgia, Mercer University, \\ School of Medicine, Macon, Georgia
}

\begin{abstract}
$\checkmark$ Herbert Olivecrona was one of the pioneers of modern neurosurgery. In one of his articles, published more than half a century ago, Olivecrona shared his thoughts on the development of neurosurgery in the first half of the twentieth century and his vision regarding the future of neurosurgery and neurosurgical training. In the present paper, the authors communicate their reflections and thoughts on Herbert Olivecrona's visionary article.
\end{abstract}

\section{Key Words - Herbert Olivecrona neurosurgical evolution}

$\mathrm{H}$ ERBERT Olivecrona (1891-1980) is considered one of the founders of modern neurosurgery. His pioneering work at the Serafimer Hospital in Stockholm (where he collaborated with Eric Lysholm, who substantially improved the diagnostic potential of ventriculography) contributed greatly to the perfection and standardization of neurosurgical techniques and treatment. In 1951 , Olivecrona wrote a visionary article on the advances in the field of neurosurgery during the nearly three decades of his career, sharing his thoughts about the future of the discipline. Having the undoubted advantage of witnessing the development of neurosurgery for more than half a century after the appearance of Olivecrona's article, we would like to communicate our reflections on the writings of this pioneer of modern neurosurgery.

In his text, after acknowledging the contribution of Harvey Cushing (1869-1939) and others to the genesis of neurosurgery, Olivecrona ${ }^{1}$ rapidly identifies what he wisely considers to be the greatest breakthrough of his time in this specialty: the design and use of the electrocautery unit and suction. Deeply aware of the importance of technology for optimal surgery, he gives the electrocautery unit and suction full credit for the control of intraoperative hemorrhage and the cleaning of the operative field, two considerations that are of paramount importance, as simple as they may seem. Indeed, mainly because of the complex anatomy and vulnerability of the tissue involved, the advancement of neurosurgery is highly dependent on the progress of technology. Who wouldn't agree that, in the years that followed Olivecrona's article, the introduction of the operating microscope and, perhaps, the stereotactic

Abbreviation used in this paper: $\mathrm{MR}=$ magnetic resonance. device invented by Lars Leksell (1907-1986), as well as the achievements in the fields of neuroanesthesiology and neurointensive care monitoring, have revolutionized neurosurgery and are to the greatest extent responsible for the dramatic improvement in surgical outcome?

Optimal surgical technique is meaningless, however, if not preceded by an accurate, or nearly accurate, localization of the lesion. Olivecrona expresses his gratitude to Walter Dandy (1886-1946) for the development of ventriculography, which in concert with angiography enabled neurosurgeons to localize and, in many instances, correctly diagnose intracranial pathological conditions. Even a neurosurgical authority of Herbert Olivecrona's stature could hardly foresee the revolution in the imaging of the central nervous system that would occur in the ensuing decades. The introduction of diagnostic modalities such as computerized tomography, positron emission tomography, and MR imaging, along with refinements in the latter (proton MR spectroscopy, diffusion- and perfusionweighted MR imaging, functional MR imaging, and diffusion tensor imaging), has had a tremendous impact on the preoperative diagnosis as well as the postoperative follow-up care of neurosurgical patients. These developments have inspired a feeling of deep respect in young neurosurgeons such as us for the heroic efforts made by neurosurgeons only a few decades ago.

In his article, Olivecrona emphasizes the new fields of neurosurgical intervention that had recently arisen. He recognizes the potential for treating functional disturbances by surgical intervention as well as the advances in surgical management of medically refractory epilepsy and extrapyramidal disorders. Special reference is made to the treatment of hypertension and angina pectoris with sympathectomy, which Olivecrona almost prophetically notes might lose its importance once medical, nonintervention- 
al treatments were developed. Following the spirit and beliefs of his time, he considers the development by Egas Moniz (1875-1955) of subcortical dissection and lobotomy for the treatment of certain psychiatric disorders as the greatest milestone in neurosurgery. He emphasizes, though, that psychiatric neurosurgery is a new area of research, "the possibilities of which have not yet been fully explored in all its ramifications."

In the years to come, lobotomy as the treatment for psychosis would fall in disrepute due to the serious and unpredictable consequences for the patient's personality. Neuroleptic drugs would prove to be an effective pharmacological treatment for many forms of schizophrenia. Nevertheless, the functional organization of the brain remains a field of intense research, in which novel neuroimaging techniques such as functional MR imaging and positron emission tomography have greatly enlarged our understanding. Functional neurosurgery has developed rapidly during the past decades; pallidotomy, first with stereotactically guided surgery and later with cryoprobe lesioning as well as deep brain stimulation targeting various anatomical areas, has been proven to alleviate parkinsonian symptoms. The surgical treatment of epilepsy has also greatly evolved; again, the contribution of modern diagnostic modalities such as MR imaging, video-electroencephalography monitoring, magnetoencephalography, ictal single-photon emission computerized tomography, subtracted ictal-interictal single-photon emission computerized tomography coregistered with magnetic resonance imaging (known as SISCOM), and stereo-electroencephalography cannot be overemphasized.

Neurosurgical education in medical schools and the training program that a young doctor should follow to become a neurosurgeon are also addressed in Olivecrona's article. He advocates an introduction to neurosurgery in the curriculum of the medical faculties, so that students will have an early exposure to this developing surgical subspecialty. It is interesting to find that the same issue is still relevant today. In most European universities, neurosurgery remains a neglected field that students have to discover alone, although almost all university hospitals nowadays house neurosurgical departments. However, the heavily loaded medical curriculum and the increased amount of knowledge that must be transmitted today, rather than ignorance or underestimation of the importance of neurosurgery, are primarily responsible.

According to Olivecrona, neurosurgical training should entail at least 2 or 3 years of general surgery and 1 to 2 years of neurology and psychiatry, before entering the main part of neurosurgical training, which should last for several years. Training in radiology and neuropathology, although desirable, is excluded from his proposed program due to the high degree of specialization in these fields. It is interesting to find that the same debate on the exact form of neurosurgical training continues in the neurosurgical community. Furthermore, the great advances in and increased number of applications for neurosurgery have raised questions about the need for further subspecialization. The continuous refinement of surgical techniques and the increase in the amount of knowledge pertinent to each field of neurosurgery seriously call into question the existence of "super-neurosurgeons."

The discussion about the need for further training in a specific neurosurgical field has multiple implications, both practical and legal. May a neurosurgeon in one of the subspecialties perform procedures and provide care for patients exceeding the strict limits of this subspecialization? For example, does a neurosurgeon who has been trained for another 2 to 3 years in pediatric neurosurgery have the right to perform spinal or cerebrovascular procedures in adult patients? Similarly, does a nonsubspecialized, general neurosurgeon have the right to perform a complex pediatric or spinal neurosurgical procedure? The role of the insurance and legal systems should not be neglected in this debate. Furthermore, if the practice of each neurosurgeon is restricted to his or her subspecialization, will there be a sufficient number of practitioners to cover the emerging neurosurgical needs of their communities?

Another controversial issue that has arisen among the supporters of subspecialization is whether this training should take place after the basic neurosurgical training, or whether we should radically modify the concept of "basic neurosurgical training" and incorporate the so-called subspecialization after the first 3 to 4 years of the neurosurgical residency program. For instance, should a young resident wishing to dedicate him- or herself to pediatric neurosurgery wait to finish a 6- to 7-year-long program, and then begin with training in his or her particular field of interest, or should this individual be given the opportunity to subspecialize in pediatric neurosurgery after the first few years of neurosurgical training?

Both sides have demonstrated strong and convincing arguments. The supporters of a long residency followed by a separate subspecialization advocate that a neurosurgeon should be able to perform a wide range of neurosurgical procedures and that the subspecialization should only involve a specific area of interest. They further support their argument by emphasizing that a young neurosurgeon should be given the opportunity to gain a wide exposure in most aspects of neurosurgery before it is demanded of him or her to choose a specific field. On the contrary, the supporters of an early subspecialization point out that with the expanding amount of knowledge in this field, it is impossible for a neurosurgeon to remain competitive in anything more than his or her particular area of interest (for example, spinal or pediatric neurosurgery). In the same way in which neurosurgery emerged as a distinct specialization in the first half of the twentieth century, they say, the various subspecializations should be integrated into the neurosurgical training programs. Furthermore, they assert that an extended training program, including 6 to 7 years of "basic" training and then 3 to 4 more years of subspecialization, might discourage young physicians from entering into neurosurgery. Which of the two opinions will prevail remains to be seen in the near future.

Olivecrona's concerns regarding standardization of the neurosurgical training in different countries, along with the creation of tighter bonds between the members of the European neurosurgical society, still lend themselves to vivid discussions. The current curriculum for neurosurgical training in Europe is not uniform, despite the indisputable steps toward a more standardized training that have taken place during the last decades. The rapid process of the political and financial unification of Europe poses further demands on the establishment of a European neurosurgical training program that guarantees the same level of 


\section{Olivecrona on the mid-20th century development of neurosurgery}

education among neurosurgeons from the different European countries. The creation of a well-structured European examination system for the accreditation of neurosurgeons, which is currently in process, constitutes a great step in that direction. Additionally, the formation of a European Society of Neurosurgery, strongly encouraged by Herbert Olivecrona almost half a century ago, is today a reality. The exchange of scientific views and achievements inside the European neurosurgical community has been a prerequisite for the development of neurosurgery. However, there is still room for further strengthening of the European Society of Neurosurgery and for better and more effective communication among its members. The multiplanar unification of Europe both requires and facilitates such a development.

Herbert Olivecrona, in his letter to Acta Neurochirurgica more than 50 years ago, shared with his colleagues his thoughts on as well as his visions of the development of neurosurgery. It is overwhelmingly interesting to acknowledge the importance of his considerations and to realize their timely nature more than half a century later. Many of his concerns and proposals could still lead, without doubt, to interesting debates on neurosurgical panels today.

\section{Reference}

1. Olivecrona H: Neurosurgery, past and present. Acta Neurochir (Wien) 2:4-8, 1951

Manuscript received February 6, 2006.

Accepted in final form April 18, 2006.

Address reprint requests to: Kostas N. Fountas, M.D., Ph.D., 840 Pine Street, Suite 880, Macon, Georgia 31201. email: knfountasmd@excite.com. 